\title{
CONTROLE QUÍMICO DA LARVA DE Diabrotica spp. NA CULTURA DO MILHO EM SISTEMA DE PLANTIO DIRETO
}

\author{
PAULO AFONSO VIANA ${ }^{1}$ E AROLDO IRIO MAROCHI ${ }^{2}$ \\ ${ }^{1}$ Pesquisador, Embrapa Milho e Sorgo. Caixa Postal 151, CEP: 35701-970 Sete Lagoas, MG. E-mail: \\ pviana@cnpms.embrapa.br (autor para correspondência) \\ ${ }^{2}$ Rod. PR 151, Km 288, Caixa Postal 1003, CEP.84166-990 Castro, PR.
}

Revista Brasileira de Milho e Sorgo, v.1, n.2, p.1-11, 2002

RESUMO - Avaliou-se a eficiência de diversos inseticidas, aplicados através de diferentes métodos, visando o controle de larvas de Diabrotica spp. na cultura do milho em sistema de plantio direto. Os ensaios foram conduzidos em oito locais na regíão de Ponta Grossa, PR, durante dois anos consecutivos. Os tratamentos constaram de diferentes inseticidas e métodos de aplicação. O delineamento experimental foi o de blocos ao acaso, com quatro repetições. Avaliou-se o número de larvas do inseto em amostras de solo, retiradas ao redor das plantas (no volume de $20 \times 20 \times 20 \mathrm{~cm}$ ). Os danos das raízes foram medidos utilizando-se uma escala de notas de 1 a 6 . Também foram avaliados estande, plantas com sintomas de ataque e rendimento de grãos. Os inseticidas chlorpyrifos $10 \mathrm{G}$, terbufos $50 \mathrm{G}$, tebupirimfos $20 \mathrm{G}$, fipronil $20 \mathrm{G}$, chlorpyrifos $450 \mathrm{SC}$ e $480 \mathrm{CE}$, aplicados no sulco de plantio, foram os que apresentaram melhor eficiência no controle das larvas de Diabrotica spp. O tratamento de sementes com thiodicarb, fipronil, imidacloprid, furathiocarb e fipronil+thiodicarb apresentaram baixo controle de larvas. Verificou-se que a proteção do sistema radicular do milho pelos inseticidas proporcionou um aumento significativo no rendimento de grãos (54\%).

Palavras-chave: insecta, inseticida, praga subterrânea, vaquinha, Zea mays.

\section{CHEMICAL CONTROL OF Diabrotica spp. LARVAE DAMAGING MAIZE UNDER NO-TILLAGE SYSTEM}

\begin{abstract}
The objective of this study was to evaluate the efficacy of insecticides application to control the corn rootworm, Diabrotica spp., on no-till corn. Field trials were conducted at eight locations in Ponta Grossa, PR, during two years. The treatments used in plots were different insecticides and application methods. The experimental design was a randomized complete block replicated four times. To evaluate the effect of insecticides, soil samples of $20 \times 20 \times 20 \mathrm{~cm}$ were taken around the corn plants and examined to recover rootworm larvae. Root systems were evaluated for rootworm damage using a 1-6 visual scale. Also, were evaluated stand, percentage of plants with symptoms and grain yield. The insecticides chlorpyrifos $10 \mathrm{G}$, terbufos $50 \mathrm{G}$, tebupirimfos $20 \mathrm{G}$, fipronil $20 \mathrm{G}$, and chlorpyrifos $450 \mathrm{SC}$ e 480 EC applied in furrow provided the best larvae control. Seed treatment with thiodicarb, fipronil, imidacloprid, furathiocarb and fipronil+thiodicarb showed low efficiency to larval control. Insecticides application prevented root damage and yield loss (54\%).
\end{abstract}

Key words: insecta, insecticide, soil insect, corn rootworm, Zea mays. 
Estima-se que, somente nos Estados Unidos, gasta-se anualmente US\$ 1 bilhão para o controle de três espécies de Diabrotica que atacam a cultura do milho e cerca de US\$ 100 milhões para o controle da praga em outras culturas (Metcalf, 1986). O gênero Diabrotica possui cerca de 338 espécies, sendo seis consideradas pragas nos trópicos. No Brasil, a espécie predominante é a Diabrotica speciosa, cujos adultos são polífagos e alimentamse das folhas de hortaliças (solanáceas, cucurbitáceas, crucíferas, gramíneas), feijoeiro, soja, girassol, bananeira, algodoeiro e milho. As larvas atacam as raízes do milho e tubérculos de batata. $\mathrm{O}$ dano causado nas raízes do milho pela larva de Diabrotica spp. interfere na absorção de nutrientes e água e reduz a estabilidade das plantas, ocasionando acamamento em situações de ventos fortes e de alta precipitação pluviométrica (Branson \& Ortman, 1971). A distribuição das larvas é em "reboleira", sendo alta a variabilidade, ocorrendo de 0 a 100 larvas por planta (Mayo, 1986). Segundo Sechriest (1969) e Bergman et al. (1981), cerca de $90 \%$ das larvas se concentram ao redor das plantas, sendo o primeiro ínstar disperso e os demais concentrados no raio de $10 \mathrm{~cm}$ em torno do sistema radicular.

O controle químico tem sido o método mais utilizado para o controle das várias espécies de Diabrotica. No Brasil, trabalhos visando o controle de larvas de D. speciosa atacando a cultura do milho são escassos, impossibilitando uma recomendação eficiente do inseticida e seu método de aplicação no controle dessa praga, enquanto que, em outros países, são abundantes as informações sobre o controle de outras espécies do gênero. De acordo com Metcalf (1986), 50 a 60\% da área plantada com milho nos Estados Unidos é tratada anualmente com inseticidas de solo para o controle de larvas de Diabrotica spp. No passado, o controle do complexo de pragas do solo era realizado eficientemente com inseticidas de longo período residual e largo espectro de ação, como os clorados. Atualmente, os inseticidas utilizados possuem curto período residual e sua ação é influenciada pela formulação, método de aplicação, incorporação e pelas condições do solo, como umidade, temperatura e presença de microorganismos (Harris, 1966 e 1967, Getzin, 1985).

A persistência dos inseticidas tem sido considerada um fator importante no controle da larva de Diabrotica. O ideal é que o inseticida persista no solo por seis a dez semanas, conferindo proteção à planta no período mais suscetível à praga (Levine \& Oloumi-Sadeghi, 1991). Os inseticidas, geralmente, são utilizados em áreas com histórico de ocorrência da praga e são aplicados sobre o sulco de plantio, em uma faixa de $15 \mathrm{~cm}$, visando proteger as plantas do ataque da larva (Sutter et al., 1989).

Estudos realizados por Sutter et al. (1991) indicaram que a taxa de sobrevivência de larvas de Diabrotica virgifera virgifera, atacando as raízes do milho, foi reduzida para menos de $50 \%$ com inseticidas aplicados na época do plantio. Brandenburg \& Herbert, Jr (1991) compararam o efeito do tratamento profilático com os inseticidas chlorpyrifos e fonofos para o controle de larvas de Diabrotica undecimpunctata howardi atacando as raízes de amendoim. Os resultados mostraram eficiência no controle da praga para os inseticidas avaliados em aplicação na área total antes do plantio, sobre a fileira na época do plantio e sobre a fileira no estádio de florescimento da cultura.

Apple et al. (1977) relataram o efeito dos inseticidas organofosforados e carbamatos sobre a produtividade de grãos, número de larvas por raiz, número de ovos por unidade de solo e danos causados às raízes. Ressaltaram que a diferença na produtividade devido ao tratamento de inseticidas foi irregular e inconsistente. Os inseticidas terbufos, 
tefluthrin, chlorpyrifos, carbofuran, fonofos, isofenphos, phorate, ethoprop e ingredientes ativos oriundos de produtos naturais têm sido avaliados para o controle da larva $D$. virgifera virgifera em milho (Sutter et al., 1989,1990; Davis \& Coleman, 1997; Lee et al., 1997).

Segundo Sutter et al. (1989), a proteção das raízes do milho proporcionada pelo tratamento com inseticida foi altamente variável, sugerindo ser dependente do ano (fatores edáficos e ambientais) e densidade populacional da praga. Os resultados obtidos por Sutter et al. (1990) indicaram que o tratamento com inseticidas reduziu significativamente o acamamento de plantas e proporcionou maior rendimento de grãos, mostrando, eficiência nos anos avaliados. Parcelas tratadas com carbofuran apresentaram maior acamamento que os demais tratamentos, enquanto que as tratadas com terbufos e isofenphos não mostraram, nos quatro anos da pesquisa, consistência nos resultados para prevenir perdas no rendimento de grãos. Davis \& Colleman (1997) verificaram que, em ensaios conduzidos no período de dois anos, inseticidas aplicados no solo (terbufos, tefluthrin e chlorpyrifos), associados com fertilizantes orgânico e inorgânico, reduziram significativamente os danos das larvas nas raízes do milho, embora o maior rendimento de grãos tenha ocorrido somente em um dos anos nas parcelas tratadas com inseticidas. Por outro lado, o maior rendimento de silagem ocorreu em ambos os anos nas parcelas tratadas.

Avaliou-se, neste trabalho, a eficiência de diversos inseticidas, aplicados através de diferentes métodos, no controle de larva de Diabrotica spp. na cultura do milho em sistema de plantio direto.

\section{Material e Métodos}

Os ensaios foram conduzidos nos campos experimentais e demonstrativos das cooperativas Batavo (Ponta Grossa), Castrolanda (Castro) e
CAPAL (Arapoti). Nesses locais, verificou-se a presença de adultos da $D$. speciosa (maioria) e de $D$. viridula. Entretanto, nas avaliações deste trabalho, as larvas dessas espécies não foram distinguidas. Visando obter infestação natural da praga para avaliar os inseticidas e respectivos métodos de aplicação, foram selecionados quatro locais no primeiro ano e dois locais no segundo ano, em áreas de plantio direto de milho na região de Ponta Grossa, PR. A cultivar de milho utilizada foi a P-3072, semeada em plantio direto, no espaçamento de $0,80 \mathrm{~m}$ entre as fileiras, visando obter o estande de 62.500 plantas ha-1. A adubação de plantio foi de $300 \mathrm{~kg} \mathrm{ha}^{-1}$ de fórmula 10-28-16 $+\mathrm{Zn}$, complementando com $160 \mathrm{~kg} \mathrm{ha}^{-1}$ de uréia em cobertura.

No primeiro ano, as parcelas foram de cinco fileiras de seis metros de comprimento para os ensaios instalados na Batavo I, Castrolanda I e Arapoti. Para os locais Batavo II e Castrolanda II, utilizaram-se parcelas com uma fileira de $20 \mathrm{~m}$ de comprimento. No segundo ano, utilizaram-se parcelas representadas por uma fileira de $25 \mathrm{~m}$.

Os inseticidas avaliados e suas respectivas doses estão apresentados nas Tabelas 1 a 5. Para sementes tratadas com inseticidas (TS), foi utilizado um frasco de dois litros e agitado durante cinco minutos. Os granulados foram distribuídos manualmente no sulco de plantio, aberto com sacho. Nos tratamentos de pulverização no sulco de plantio, utilizouse um equipamento costal pressurizado a gáscarbônico, com ponta de jato plano 80.02EVS, na pressão de 221 pol$^{-2}$, altura de $12 \mathrm{~cm}$ em relação ao sulco, com deposição em faixa efetiva de $13 \mathrm{~cm}$ de largura e volume de 1001 ha $^{-1}$. Após a aplicação dos inseticidas, fechou-se o sulco e plantou-se o milho com matraca manual. O delineamento experimental foi em blocos ao acaso com quatro repetições.

Considerou-se a principal variável a ser analisada a contagem do número de larvas em amostras 
de solo (volume: $20 \times 20 \times 20 \mathrm{~cm}$ ) retiradas em torno de cada planta de milho. O solo foi peneirado e os insetos coletados foram armazenados em frascos com álcool a 70\%. Após a retirada do solo das raízes, estas foram colocadas em recipientes contendo água, para limpeza e retirada de larvas presentes nas suas partes internas. As raízes foram submetidas a análise de danos, utilizando-se uma escala de 1 a 6, proposta por Hills \& Peters (1971). Nessa escala, 1 corresponde a nenhum dano ou somente alguns sinais de alimentação nas raízes; 2 , sinais de alimentação evidente, sem apresentar raízes destruídas numa extensão de 4 cm da planta; 3, várias raízes destruídas numa extensão de $4 \mathrm{~cm}$ da planta, mas sem apresentar um nódulo de raízes inteiramente destruído; 4 , um nódulo de raízes inteiramente destruído; 5 , dois nódulos de raízes inteiramente destruídos e 6 , três ou mais nódulos de raízes inteiramente destruídos. De acordo com Mayo (1986), essa escala é considerada padrão para a maioria dos trabalhos de avaliação de inseticidas para o controle da larva de Diabrotica spp. As amostragens de solo foram realizadas em 20 plantas por parcela, no primeiro ano, e 15 plantas nos ensaios do segundo ano. As avaliações foram realizadas 47 dias após o plantio e variaram para cada local, de acordo com as condições de precipitação pluviométrica, para que o solo pudesse ser peneirado. Outros parâmetros avaliados foram: estande, plantas anormais e rendimento de grãos na colheita. Os dados obtidos foram submetidos à análise de variância (teste $\mathrm{F} \leq 5 \%$ ) e as médias, comparadas através do teste de Tukey, ao nível de 5\% de probabilidade.

\section{Resultados e Discussão}

No período em que os experimentos foram conduzidos, em cada ano, a infestação foi mais alta somente em um local, nos demais a infestação foi moderada. A análise conjunta dos dados para ano mostrou que alguns inseticidas tiveram comportamento diferente de acordo com o local, procedendo-se, assim, à comparação de médias pelo teste de Tukey para cada local. Nos anos considerados, a maior infestação da praga ocorreu em Castrolanda, onde o número médio de larvas por planta excedeu a quatro (Tabela 1). Os inseticidas chlorpyrifos 10 $\mathrm{G}$, terbufos $50 \mathrm{G}$, tebupirimfos $20 \mathrm{G}$, fipronil $20 \mathrm{G}$, na formulação granulada, aplicada no sulco de plantio, foram, em ambos os anos, os que apresentaram os menores números de larvas de Diabrotica spp. Inseticidas pulverizados no sulco de plantio, como o chlorpyrifos $450 \mathrm{SC}$ e $480 \mathrm{CE}$, também apresentaram boa performance no controle da praga, embora não tenham mostrado boa eficiência no primeiro ano em Castrolanda.

Embora o tratamento de sementes seja um método fácil e barato de se utilizar, observou-se baixa eficiência no controle de larvas dessa praga. A melhor formulação foi a granulada, aplicada no sulco de plantio. Mayo (1986) relatou que a maioria dos trabalhos visando o controle de Diabrotica resulta numa informação da performance relativa de um inseticida comparado com outro em diferentes locais. Nesse caso, a eficiência do inseticida é influenciada pela época de aplicação, umidade, microorganismos e fatores edáficos. Segundo o autor, os resultados geralmente diferenciam os inseticidas que proporcionam excelente controle da larva daqueles de baixo controle. Entretanto, torna-se difícil identificar os inseticidas que apresentam níveis intermediários de controle.

A avaliação dos danos causados pelas larvas de Diabrotica nas raízes mostrou boa relação com o controle apresentado pelos inseticidas, através do número de larvas sobreviventes. Verificaramse menores danos no sistema radicular nas parcelas tratadas com os inseticidas mais efetivos na redução do número de larvas (Tabela 2). Segundo Turpin et al. (1972) e Mayo (1986), para D. virgifera 
virgifera, danos nas raízes excedendo a 2,5-3,0 têm sido utilizados para indicar perda econômica para a cultura de milho visando a colheita de grãos. Resultados obtidos mais recentemente por Sutter et al. (1990) sustentam que a aplicação de inseticidas é justificável quando o dano nas raízes alcança valores entre 4,0 e 5,0. Davis (1994) concluiu que índices mais conservadores de danos (2,5-3,0), são mais indicados para cultura com maior valor agregado, como silagem. Weiss \& Mayo (1983) encontraram em amostragem de campo, para larva de Diabrotica spp., que cerca de 1,8 larva por planta representou dano de 2,7 em uma escala de 1-6. Entretanto, o dano econômico para a lavoura somente foi verificado para valores acima de 3,40 , onde a população foi de cinco larvas por planta. Na Tabela 2, pode-se observar que os danos com notas ao redor de quatro ocorreram nas parcelas testemunha de quatro locais, nas quais foram observadas, em média, mais de duas larvas por planta. Parcelas tratadas com chlorpyrifos $10 \mathrm{G}$, fipronil $20 \mathrm{G}$, terbufos $50 \mathrm{G}$, no sulco de plantio, apresentaram reduzido número de larvas e menor dano no sistema radicular. O chlorpyrifos $450 \mathrm{SC}$ e $480 \mathrm{CE}$, pulverizados no sulco de plantio, mostraram boa performance na maioria dos locais onde os ensaios foram conduzidos, excetuando-se Castrolanda, no primeiro ano.

TABELA 1. Número médio de larvas de Diabrotica spp. por planta de milho, nos diferentes tratamentos ${ }^{1,2}$.

\begin{tabular}{|c|c|c|c|c|c|c|c|}
\hline \multirow{2}{*}{ Inseticidas/ Formulação } & \multirow{2}{*}{$\begin{array}{l}\text { Dose } \\
\left(\mathrm{g} \text { i.a. ha }{ }^{-1}\right)\end{array}$} & \multicolumn{2}{|c|}{$\mathbf{L}_{1}$} & \multicolumn{2}{|c|}{$\mathbf{L}_{2}$} & \multirow{2}{*}{$\begin{array}{r}L_{3} \\
\text { Ano I }\end{array}$} & \multirow{2}{*}{$\begin{array}{r}\mathbf{L}_{4} \\
\text { Ano I }\end{array}$} \\
\hline & & Ano I & Ano II & Ano I & Ano II & & \\
\hline Testemunha & $\cdots$ & $1,7 \mathrm{ab}$ & $4,4 \mathrm{a}$ & $2,1 \mathrm{a}$ & $2,4 \mathrm{a}$ & $6,4 a$ & $1,4 \mathrm{n} . \mathrm{s}$ \\
\hline Chlorpyrifos $/ 450 \mathrm{SC}$ & 1.125 & $0,2 \mathrm{~d}$ & $0,2 \mathrm{~d}$ & 1,1abc & 0,2 bodef & $2,0 \mathrm{fgh}$ & 0,6 \\
\hline Chlorpyrifos/480 CE & 1.200 & $0,2 \mathrm{~d}$ & $0,5 \mathrm{~cd}$ & $0,7 \mathrm{bc}$ & $0,1 \mathrm{ef}$ & $3,8 \mathrm{cdf}$ & 0,2 \\
\hline Chlorpyrifos/10 G & 1.100 & $0,1 \mathrm{~d}$ & $0,6 \mathrm{bcd}$ & $0,2 \mathrm{c}$ & $0,0 \mathrm{e}$ & $0,5 \mathrm{~h}$ & 0,4 \\
\hline Thiodicarb/350 TS & $700^{3}$ & $1,2 \mathrm{abc}$ & $2,9 \mathrm{ab}$ & $1,5 \mathrm{ab}$ & 0,5 bcdef & $6,2 \mathrm{ab}$ & 0,5 \\
\hline Fipronil/20 G & 100 & $0,2 \mathrm{~d}$ & 0,6 bod & $0,2 \mathrm{c}$ & 0,2 odef & $1,0 \mathrm{gh}$ & 0,4 \\
\hline Fipronil/250 TS & $500^{3}$ & $0,8 \mathrm{bcd}$ & 1,1abcd & $0,7 \mathrm{bc}$ & $1,0 \mathrm{ab}$ & $2,5 \mathrm{efg}$ & 0,3 \\
\hline Phorate/50 G & 2.500 & $0,8 \mathrm{bcd}$ & 1,9abcd & $0,6 \mathrm{bc}$ & $0,8 \mathrm{bcd}$ & $0,4 \mathrm{~h}$ & 0,2 \\
\hline Terbufos/50 G & 1.500 & $0,4 \mathrm{~cd}$ & $1,7 \mathrm{abcd}$ & $0,1 \mathrm{c}$ & 0,2 bedef & $0,5 \mathrm{~h}$ & 0,6 \\
\hline Terbufos/50 G & 2.000 & $0,2 \mathrm{~d}$ & $0,9 \mathrm{bcd}$ & $0,1 \mathrm{c}$ & 0,3 bcdef & $0,3 \mathrm{~h}$ & 0,2 \\
\hline Imidacloprid/700 PM & $350^{3}$ & $2,0 \mathrm{a}$ & & $2,0 \mathrm{a}$ & & $5,3 \mathrm{abc}$ & 0,6 \\
\hline Imidacloprid/700 PM & $140^{3}$ & $1,4 a b$ & & $1,2 \mathrm{abc}$ & & $4,6 \mathrm{abcd}$ & 0,8 \\
\hline Tebupirimfos $/ 20 \mathrm{G}$ & 125 & $0,2 \mathrm{~d}$ & $0,7 \mathrm{bod}$ & $0,2 \mathrm{c}$ & 0, lef & $0,8 \mathrm{gh}$ & 0,2 \\
\hline Furathiocarb/400 SC & $640^{3}$ & $1,0 \mathrm{bcd}$ & & $2,1 \mathrm{a}$ & & 3,1def & 1,2 \\
\hline Isazofos/50 SC & 1.500 & $0,8 \mathrm{bcd}$ & & $0,7 \mathrm{bc}$ & & 1,1gh & 0,5 \\
\hline Isazofos $/ 50 \mathrm{SC}$ & $1.500^{4}$ & $1,4 a b$ & & $1,2 \mathrm{abc}$ & & 4,3 bcde & 1,3 \\
\hline Carbofuran $/ 50 \mathrm{G}$ & 1.500 & $1,6 a b$ & $2,3 \mathrm{abc}$ & $2,2 \mathrm{a}$ & 0,6 bcdef & $4,5 \mathrm{bcd}$ & 1,3 \\
\hline Tebupirimfos/20 G & 160 & & $0,4 \mathrm{~cd}$ & & $0,0 \mathrm{f}$ & & \\
\hline Tebupirimfos/20 G & 200 & & $0,3 \mathrm{~cd}$ & & 0, lef & & \\
\hline Isazofos/50 SC & 500 & & $2,2 \mathrm{abc}$ & & 0,6 bedef & & \\
\hline Isazofos/50 SC & 750 & & 1,6abcd & & $0,9 \mathrm{bc}$ & & \\
\hline Isazofos/50 SC & 1.000 & & 1,9abcd & & 0,8 bode & & \\
\hline Fipronil+thiodicarb $/ 250+300 \mathrm{TS}$ & $500+360^{3}$ & & $2,2 \mathrm{abcd}$ & & 0,6 bcdef & & \\
\hline C.V. $(\%)$ & & 39,61 & 33,5 & 41,09 & 32,3 & 21,42 & 30,46 \\
\hline
\end{tabular}

${ }^{1}$ Dados originais foram transformados para $\sqrt{ } \mathrm{X}+0,5$ para análise estatística.

${ }^{2}$ Médias seguidas da mesma letra, na coluna, não diferem significativamente, pelo teste de Tukey a $5 \%$.

${ }^{3} \mathrm{~g}$ i.a. $100 \mathrm{Kg}^{-1}$ sementes.

${ }^{4}$ Pulverização total da área.

Ano I: $\mathrm{L}_{1}, \mathrm{~L}_{2}=$ Batavo I e II, $\mathrm{L}_{3}=$ Castrolanda, $\mathrm{L}_{4}=$ Arapoti; Ano II: $\mathrm{L}_{1}=$ Castrolanda, $\mathrm{L}_{2}=$ Batavo. 
TABELA2. Média dos danos de larvas de Diabrotica spp. nas raízes de milho, nos diferentes tratamentos ${ }^{1,2}$.

\begin{tabular}{|c|c|c|c|c|c|c|c|}
\hline \multirow{2}{*}{ Inseticidas / Formulação } & \multirow{2}{*}{$\begin{array}{l}\text { Dose } \\
\left(\mathrm{g} \text { i.a. } \mathrm{ha}^{-1}\right)\end{array}$} & \multicolumn{2}{|c|}{$\mathbf{L}_{1}$} & \multicolumn{2}{|c|}{$\mathbf{L}_{2}$} & \multirow{2}{*}{$\begin{array}{r}\mathbf{L}_{3} \\
\text { Ano I }\end{array}$} & \multirow{2}{*}{$\begin{array}{r}\mathrm{L}_{4} \\
\text { Ano I }\end{array}$} \\
\hline & & Ano I & Ano II & Ano I & Ano II & & \\
\hline Testemunha & -- & $3,1 \mathrm{ab}$ & $3,9 \mathrm{a}$ & $4,2 \mathrm{a}$ & $4,0 \mathrm{a}$ & $4,9 \mathrm{a}$ & $3,0 \mathrm{ab}$ \\
\hline Chlorpyrifos/450 SC & 1.125 & $1,9 \mathrm{e}$ & $1,5 \mathrm{e}$ & $2,7 \mathrm{defgh}$ & $2,3 \mathrm{efg}$ & 3,1 def & 2,0def \\
\hline Chlorpyrifos/480 CE & 1.200 & $1,9 \mathrm{e}$ & $1,7 \mathrm{de}$ & $2,7 \mathrm{defgh}$ & $2,3 \mathrm{efg}$ & $2,4 \mathrm{efg}$ & $1,7 \mathrm{ef}$ \\
\hline Chlorpyrifos/10 G & 1.100 & $1,8 \mathrm{c}$ & $1,8 \mathrm{de}$ & $1,8 \mathrm{i}$ & 2,3defg & $2,1 \mathrm{~g}$ & $1,5 \mathrm{f}$ \\
\hline Thiodicarb/350 TS & $700^{3}$ & $3,7 \mathrm{a}$ & $3,3 a b c$ & $3,4 \mathrm{bcd}$ & $3,5 \mathrm{ab}$ & $4,7 \mathrm{a}$ & $2,9 \mathrm{ab}$ \\
\hline Fipronil/20 G & 100 & $2,0 \mathrm{e}$ & $2.2 \mathrm{cde}$ & $2,0 \mathrm{hi}$ & 2,5 cdefg & $2,2 \mathrm{fg}$ & $1,5 \mathrm{f}$ \\
\hline Fipronil/250 TS & $500^{3}$ & $2,8 \mathrm{bcd}$ & 2,3 cde & 3,1 cdef & 3,3 bcd & 3,1 def & $2,4 \mathrm{bcd}$ \\
\hline Phorate/50 G & 2.500 & $2,4 \mathrm{cde}$ & $3,3 a b c$ & 2,7 defgh & $3,0 \mathrm{cdefg}$ & $2,2 \mathrm{fg}$ & $1,9 \mathrm{def}$ \\
\hline Terbufos $/ 50 \mathrm{G}$ & 1.500 & $2,2 \mathrm{de}$ & 2,5 bcde & 2,5 efgh & 2,5 cdefg & $2,0 \mathrm{~g}$ & $1,7 \mathrm{ef}$ \\
\hline Terbufos $/ 50 \mathrm{G}$ & 2.000 & $1,9 \mathrm{e}$ & $1,6 \mathrm{de}$ & 2,1 ghi & 2,5 cdefg & $1,9 \mathrm{~g}$ & $1,5 \mathrm{ef}$ \\
\hline Imidacloprid/700 PM & $350^{3}$ & $3,0 \mathrm{abc}$ & & 3,3 bcde & & 3,4 cde & 2,2 cde \\
\hline Imidacloprid/700 PM & $140^{3}$ & $3,1 \mathrm{ab}$ & & $2,5 \mathrm{efgh}$ & & $3,6 \mathrm{bod}$ & $3,0 \mathrm{ab}$ \\
\hline Tebupirimfos $/ 20 \mathrm{G}$ & 125 & $2,1 \mathrm{e}$ & $1,8 \mathrm{de}$ & $2,4 \mathrm{fghi}$ & $2,0 \mathrm{fg}$ & $1,9 \mathrm{~g}$ & $1,5 \mathrm{f}$ \\
\hline Furathiocarb/400 SC & $640^{3}$ & $3,2 \mathrm{ab}$ & & $4,0 \mathrm{ab}$ & & $4,4 a b c$ & $3,1 \mathrm{a}$ \\
\hline Isazofos $/ 50 \mathrm{SC}$ & 1.500 & $2,3 \mathrm{de}$ & & 2,9defg & & $2,2 \mathrm{fg}$ & $1,7 \mathrm{ef}$ \\
\hline Isazofos $/ 50 \mathrm{SC}$ & $1.500^{4}$ & $3,6 a$ & & $3,4 a b o d$ & & 3,5 bod & $2,9 \mathrm{ab}$ \\
\hline Carbofuran/50 G & 1.500 & $3,5 \mathrm{a}$ & 3,6ab & $3,8 a b c$ & $3,5 \mathrm{ab}$ & $4,5 \mathrm{ab}$ & $2,7 \mathrm{abc}$ \\
\hline Tebupirimfos/20 G & 160 & & 1,9de & & $1,8 \mathrm{~g}$ & & \\
\hline Tebupirimfos $/ 20 \mathrm{G}$ & 200 & & $1.6 \mathrm{de}$ & & $2,0 \mathrm{fg}$ & & \\
\hline Isazofos $/ 50 \mathrm{SC}$ & 500 & & $3,2 \mathrm{abc}$ & & $3,3 a b c$ & & \\
\hline Isazofos $/ 50 \mathrm{SC}$ & 750 & & $2,6 \mathrm{bcd}$ & & 3,0 bode & & \\
\hline Isazofos/50 SC & 1.000 & & 2,5 bcde & & 3,0 bode & & \\
\hline Fipronil+thiodicarb/250+300 TS & $500+360^{3}$ & & $2,5 \mathrm{cde}$ & & 2,8 bode & & \\
\hline C.V. $(\%)$ & & 7,19 & 7,3 & 8,43 & 5,2 & 9,58 & 5 \\
\hline
\end{tabular}

${ }^{1}$ Dados originais foram transformados para $\sqrt{X}+0,5$ para análise estatística.

${ }^{2}$ Médias seguidas da mesma letra, na coluna, não diferem significativamente, pelo teste de Tukey a 5\%.

${ }^{3} \mathrm{~g}$ i.a. $100 \mathrm{Kg}^{-1}$ sementes.

${ }^{4}$ Pulverização total da área.

Ano I: $\mathrm{L}_{1}, \mathrm{~L}_{2}=$ Batavo I e II, $\mathrm{L}_{3}=$ Castrolanda, $\mathrm{L}_{4}=$ Arapoti; Ano II: $\mathrm{L}_{1}=$ Castrolanda, $\mathrm{L}_{2}=$ Batavo.

Analisando-se a percentagem de plantas com sintoma de ataque (Tabela 3 ), pode-se observar que houve diferença significativa somente em três locais, sendo dois os que apresentaram o maior número de larvas por planta. Em locais como Arapoti e Batavo I, onde os solos eram mais pobres, foi observada elevada percentagem de plantas com sintomas de ataque, com baixo número de larvas. Uma possível explicação para esse fato é que as plantas sofreram mais o estresse causado pelas larvas sob essa condição. Em Arapoti, a percentagem de plantas com sintomas de ataque atingiu a 38,2\% na parcela testemunha. Em Batavo I, não foi observada consistência entre o número médio de larvas por planta, danos nas raízes e percentagem de plantas com sintoma de ataque. Nos demais locais, constatou-se uma tendência de menor percentagem de plantas atacadas para a maioria das parcelas tratadas com inseticidas. De uma maneira geral, nota-se que nos tratamentos onde foram observados o maior número de larvas, foram os que apresentaram os maiores dano no sistema radicular. Contudo, esse dano não refletiu diretamente na percentagem de plantas com sintomas de ataque (pescoço de ganso). Esse fato deve-se principalmente à distribuição de chuvas no período de condução de cada ensaio e às 
TABELA 3. Percentagem de plantas com sintoma de ataque de larva de Diabrotica spp., nos diferentes tratamentos ${ }^{1,2}$.

\begin{tabular}{|c|c|c|c|c|c|c|c|}
\hline \multirow{2}{*}{ Inseticidas/ Formulação } & \multirow{2}{*}{$\begin{array}{l}\text { Dose } \\
\left(\mathrm{g} \text { i.a. } \mathrm{ha}^{-1}\right)\end{array}$} & \multicolumn{2}{|c|}{$\mathbf{L}_{1}$} & \multicolumn{2}{|c|}{$\mathbf{L}_{2}$} & \multirow{2}{*}{$\begin{array}{c}\mathbf{L}_{3} \\
\text { Ano I }\end{array}$} & \multirow{2}{*}{$\begin{array}{c}\mathbf{L}_{4} \\
\text { Ano II }\end{array}$} \\
\hline & & Ano I & Ano II & Ano I & Ano II & & \\
\hline Testemunha & -- & 34,8 n.s. & $13,6 \mathbf{a}$ & $20,6 \mathrm{n} . \mathrm{s}$. & $14, \ln . s$. & $20,7 \mathrm{a}$ & $38,2 \mathrm{a}$ \\
\hline Chlorpyrifos/450 SC & 1.125 & 23,7 & $6,2 \mathrm{ab}$ & 10,7 & 7,1 & 10,4 cdef & $10,8 \mathrm{bc}$ \\
\hline Chlorpyrifos/480 CE & 1.200 & 30,1 & $4,8 \mathrm{~b}$ & 18,0 & 7,4 & 9,8 odef & $9,1 \mathrm{c}$ \\
\hline Chlorpyrifos/10 G & 1.100 & 21,8 & $5,2 \mathrm{~b}$ & 13,5 & 9,4 & $10,4 \mathrm{cdef}$ & $10,8 \mathrm{bc}$ \\
\hline Thiodicarb/350 TS & $700^{3}$ & 29,5 & $6,1 \mathrm{ab}$ & 18,6 & 6,2 & $16,3 \mathrm{abc}$ & $17,4 \mathrm{bc}$ \\
\hline Fipronil/20 G & 100 & 28,6 & $9,2 \mathrm{ab}$ & 13,3 & 9,0 & $7,4 \mathrm{ef}$ & $17,5 \mathrm{bc}$ \\
\hline Fipronil/250 TS & $500^{3}$ & 35,2 & $6,3 \mathrm{ab}$ & 12.5 & 8.4 & $11.8 \mathrm{cdef}$ & $21.2 \mathrm{~b}$ \\
\hline Phorate/50 G & 2.500 & 33,1 & $11,0 \mathrm{ab}$ & 19,7 & 10,4 & $11,2 \mathrm{cdef}$ & $14,3 \mathrm{bc}$ \\
\hline Terbufos $/ 50 \mathrm{G}$ & 1.500 & 36,8 & $8,3 \mathrm{ab}$ & 20,1 & 7,3 & 10, lodef & $10,7 \mathrm{bc}$ \\
\hline Terbufos $/ 50 \mathrm{G}$ & 2,000 & 27,4 & $10,8 \mathrm{ab}$ & 16,7 & 6,6 & $7,0 \mathrm{ef}$ & $10,4 \mathrm{bc}$ \\
\hline Imidacloprid/700 PM & $350^{3}$ & 33,1 & & 15,8 & & $13,3 \mathrm{bcd}$ & $9,8 \mathrm{bc}$ \\
\hline Imidacloprid/700 PM & $140^{3}$ & 35,7 & & 18,5 & & $11,7 \mathrm{bcde}$ & $13,5 \mathrm{bc}$ \\
\hline Tebupirimfos/20 G & 125 & 34,0 & $6,4 a b$ & 11,7 & 7,6 & $11,9 \mathrm{bcde}$ & $12,6 \mathrm{bc}$ \\
\hline Furathiocarb/400 SC & $640^{3}$ & 34,4 & & 13,8 & & $20,8 \mathrm{ab}$ & $23,3 \mathrm{~b}$ \\
\hline Isazofos $/ 50 \mathrm{SC}$ & $1.500^{4}$ & 28,0 & & 12,1 & & $5,9 \mathrm{f}$ & $9,9 \mathrm{c}$ \\
\hline Isazofos/50 SC & 1.500 & 27,0 & & 15,8 & & $13,0 \mathrm{bce}$ & $8,9 \mathrm{c}$ \\
\hline Carbofuran/50 G & 1.500 & 32,2 & 9,1ab & 15,8 & 6,9 & $8,9 \mathrm{def}$ & $8,1 \mathrm{c}$ \\
\hline Tebupirimfos/20 G & 160 & & $5,8 \mathrm{ab}$ & & 7,8 & & \\
\hline Tebupirimfos/20 G & 200 & & 7,3ab & & 6,3 & & \\
\hline Isazofos $/ 50 \mathrm{SC}$ & 500 & & $8,8 \mathrm{ab}$ & & 6,1 & & \\
\hline Isazofos $/ 50 \mathrm{SC}$ & 750 & & $9,2 \mathrm{ab}$ & & 7,8 & & \\
\hline Isazofos $/ 50 \mathrm{SC}$ & 1.000 & & $5,2 \mathrm{~b}$ & & 7,8 & & \\
\hline Fipronil+thiodicarb/250+300 TS & $500+360^{3}$ & & $7,9 \mathrm{ab}$ & & 5,9 & & \\
\hline C.V. $(\%)$ & & 15,30 & 18,9 & 18,63 & 32,5 & 18,5 & 22,96 \\
\hline
\end{tabular}

${ }^{1}$ Dados originais foram transformados para $\sqrt{ } \mathrm{X}+0,5$ para análise estatística.

${ }^{2}$ Médias seguidas da mesma letra, na coluna, não diferem significativamente, pelo teste de Tukey a 5\%.

${ }^{3} \mathrm{~g}$ i.a. $100 \mathrm{Kg}^{-1}$ sementes.

${ }^{4}$ Pulverização total da área.

Ano I: $\mathrm{L}_{1}, \mathrm{~L}_{2}=$ Batavo I e II, $\mathrm{L}_{3}=$ Castrolanda, $\mathrm{L}_{4}=$ Arapoti; Ano II: $\mathrm{L}_{1}=$ Castrolanda, $\mathrm{L}_{2}=$ Batavo.

características físico-química do solo da área. Geralmente, período mais longo de chuvas permite a renovação do sistema radicular, possibilitando maior sustentação das plantas de milho.

A avaliação de estande na colheita mostrou diferença significativa entre os tratamentos somente no primeiro ano (Tabela 4). No segundo ano, embora o estande das parcelas testemunhas tenha estado entre os menores, este não diferiu dos demais tratamentos. Nos ensaios, foi observado que os ataques das larvas de Diabrotica spp. ocorreram após a implantação da cultura e não foram responsáveis pela redução no estande. Possivelmente, outras espécies de insetos-pragas iniciais da cultura do milho também podem ter contribuído para reduzir o número de plantas. O dano que é causado pela alimentação da larva de Diabrotica spp. no sistema radicular dificulta a sustentação das plantas, a colheita mecânica e a absorção de nutrientes na época de enchimento de grãos (Branson \& Ortman, 1971).

Os resultados de rendimento de grãos (Tabela 5) mostraram que, de maneira geral, as parcelas testemunhas foram as que produziram menos. Os menores rendimentos de grãos foram observados no primeiro ano, em locais onde ocorreram os maiores estresses hídricos. Nesses locais, houve uma 
TABELA 4. Número de plantas por hectare em parcelas, submetidas a diferentes tratamentos, para o controle de larvas de Diabrotica spp. ${ }^{1,2}$.

\begin{tabular}{|c|c|c|c|c|c|c|c|}
\hline \multirow{2}{*}{ Inseticidas/ Formulação } & \multirow{2}{*}{$\begin{array}{l}\text { Dose } \\
\left(\mathrm{g} \text { i.a. } \mathrm{ha}^{-1}\right)\end{array}$} & \multicolumn{2}{|c|}{$\mathbf{L}_{1}$} & \multicolumn{2}{|c|}{$\mathbf{L}_{2}$} & \multirow{2}{*}{$\mathbf{L}_{3}$} & \multirow{2}{*}{$\begin{array}{r}\mathbf{L}_{4} \\
\text { Ano I }\end{array}$} \\
\hline & & Ano I & Ano II & Ano I & Ano II & & \\
\hline Testemunha & $-\ldots$ & $42688 \mathrm{f}$ & 58000 n.s. & $53125 \mathrm{ef}$ & $55500 \mathrm{n} . \mathrm{s}$. & 55188 de & $40114 d$ \\
\hline Chlorpyrifos/450 SC & 1.125 & 48750 abodef & 60150 & $55469 \mathrm{cdef}$ & 59688 & $59063 \mathrm{abc}$ & $52614 a b$ \\
\hline Chlorpyrifos/480 CE & 1.200 & 49875abode & 60250 & 54688 def & 63438 & $60500 \mathrm{ab}$ & $5000 \mathrm{~b}$ \\
\hline Chlorpyrifos/10 G & 1.100 & $44375 \mathrm{ef}$ & 57650 & $55469 \mathrm{cdef}$ & 60000 & 57063 bode & $47500 \mathrm{bc}$ \\
\hline Thiodicarb/350 TS & $700^{3}$ & $52500 \mathrm{abc}$ & 56150 & 5703 lbcde & 58625 & $54875 \mathrm{c}$ & $48864 \mathrm{bc}$ \\
\hline Fipronil/20 G & 100 & $47063 \mathrm{abcdef}$ & 59650 & $56250 \mathrm{cdef}$ & 59250 & 57813 abcde & $48636 b c$ \\
\hline Fipronil/250 TS & $500^{3}$ & $51438 \mathrm{abcd}$ & 55400 & $59844 a b c$ & 65813 & $55500 \mathrm{cde}$ & $47159 b c$ \\
\hline Phorate/50 G & 2.500 & 47813abodef & 58000 & $53906 \mathrm{ef}$ & 60000 & 57375 bode & $51477 \mathrm{ab}$ \\
\hline Terbufos/50 G & 1.500 & 47188abodef & 59000 & 54375 ef & 63938 & 57500 bode & $50909 \mathrm{ab}$ \\
\hline Terbufos/50 G & 2.000 & $50813 \mathrm{abod}$ & 57000 & $56250 \mathrm{cdef}$ & 64375 & 57688 bode & $49205 b c$ \\
\hline Imidacloprid/700 PM & $350^{3}$ & $52813 a b$ & & 59375abcd & & $58938 \mathrm{abcd}$ & $57727 \mathrm{a}$ \\
\hline Imidacloprid/700 PM & $140^{3}$ & $53000 \mathrm{a}$ & & $61719 \mathrm{ab}$ & & $61563 a$ & $50341 \mathrm{~b}$ \\
\hline Tebupirimfos $/ 20 \mathrm{G}$ & 125 & 47813abcdef & 56650 & 57500 abcde & 59875 & 57813 abcde & $49773 \mathrm{bc}$ \\
\hline Furathiocarb/400 SC & $640^{3}$ & $51750 \mathrm{abcd}$ & & $62188 \mathrm{a}$ & & $49063 \mathrm{f}$ & $42955 \mathrm{~cd}$ \\
\hline Isazofos/50 SC & 1.500 & $46438 \mathrm{cdef}$ & & $51563 f$ & & $56563 \mathrm{cde}$ & $49432 \mathrm{bc}$ \\
\hline Isazofos/50 SC & $1.500^{4}$ & 46125 def & & 54375 ef & & 56875 bode & $50909 \mathrm{ab}$ \\
\hline Carbofuran $/ 50 \mathrm{G}$ & 1.500 & 46563 bcdef & 58000 & $54375 \mathrm{cf}$ & 61875 & $54875 \mathrm{e}$ & $48864 \mathrm{bc}$ \\
\hline Tebupirimfos $/ 20 \mathrm{G}$ & 160 & & 56250 & & 62500 & & \\
\hline Tebupirimfos $/ 20 \mathrm{G}$ & 200 & & 58150 & & 62188 & & \\
\hline Isazofos/50 SC & 500 & & 58750 & & 59063 & & \\
\hline Isazofos/50 SC & 750 & & 53250 & & 62688 & & \\
\hline Isazofos/50 SC & 1.000 & & 60150 & & 60000 & & \\
\hline Fipronil+thiodicarb/ $250+300 \mathrm{TS}$ & $500+360^{3}$ & & 56750 & & & & \\
\hline C.V. $(\%)$ & & 9,1 & 5,1 & 6,0 & 9,9 & 4,7 & 9,9 \\
\hline
\end{tabular}

${ }^{1}$ Dados originais foram transformados para $\sqrt{ } \mathrm{X}+0,5$ para análise estatística.

${ }^{2}$ Médias seguidas da mesma letra, na coluna, não diferem significativamente, pelo teste de Tukey a 5\%.

${ }^{3} \mathrm{~g}$ i.a. $100 \mathrm{Kg}^{-1}$ sementes.

${ }^{4}$ Pulverização total da área.

Ano I: $\mathrm{L}_{1}, \mathrm{~L}_{2}=$ Batavo I e II, $\mathrm{L}_{3}=$ Castrolanda, $\mathrm{L}_{4}=$ Arapoti; Ano II: $\mathrm{L}_{1}=$ Castrolanda, $\mathrm{L}_{2}=$ Batavo.

maior diferenciação entre os tratamentos. O rendimento foi $32 \%$ inferior ao tratamento com o inseticida terbufos no controle da larva, em Castrolanda, e de até 54\% em Arapoti. Para os locais com alta produtividade, essa diferença não foi tão evidente, mostrando, entretanto, a tendência de maior produtividade nas parcelas onde os inseticidas tiveram melhor performance no controle das larvas. Isso indica a importância do controle da praga, principalmente em áreas sob estresses, onde os prejuízos para o agricultor são maiores, o que está de acordo com Branson et al. (1980), quando afirmaram que as perdas causadas pelas larvas de Diabrotica são consideravelmente mais elevadas quando a cultura do milho está sujeita a estresse hídrico do que em condições ideais para o seu desenvolvimento. De acordo com Mayo (1986), embora a produção seja o fator mais importante na avaliação da resposta do inseticida para o controle da larva de Diabrotica, a produtividade pode ser influenciada por diversos fatores ambientais, biológicos e agronômicos, tornando-se difícil de ser associada ao nível econômico de dano. Ainda segundo o autor, a relação entre rendimento de grãos e danos nas raízes é bastante variável. Branson et al. (1980) encontraram uma correlação de 0,54 para danos nas raízes e rendimento de grãos. Demostraram, também, que uma nota de dano nas raízes de dois, numa escala de seis pontos, resultou em aproximadamente $4 \%$ na redução da produtividade. No caso, a nota de dano está abaixo 
TABELA 5. Rendimento de grãos $\left(\mathrm{kg} \mathrm{ha}^{-1}\right)$ em parcelas tratadas com inseticidas, para o controle de larva de Diabrotica spp. ${ }^{1,2}$

\begin{tabular}{|c|c|c|c|c|c|c|c|}
\hline \multirow{2}{*}{ Inseticidas/ Formulação } & \multirow{2}{*}{$\begin{array}{l}\text { Dose } \\
\left(\mathrm{g} \text { i.a. ha } \mathrm{h}^{-1}\right)\end{array}$} & \multicolumn{2}{|r|}{$\mathbf{L}_{1}$} & \multicolumn{2}{|c|}{$\mathbf{L}_{2}$} & \multirow{2}{*}{$\begin{array}{c}\mathrm{L}_{3} \\
\text { Ano I }\end{array}$} & \multirow{2}{*}{$\begin{array}{r}\mathrm{L}_{4} \\
\text { Ano I }\end{array}$} \\
\hline & & Ano I & Ano II & Ano I & Ano II & & \\
\hline Testemunha & -- & 5335 n.s. & $6503 \mathrm{e}$ & $5795 \mathrm{~d}$ & 4891 n.s. & $2523 \mathrm{~g}$ & $2621 \mathrm{e}$ \\
\hline Chlorpyrifos $/ 450 \mathrm{SC}$ & 1.125 & 6347 & $7697 \mathrm{ab}$ & $7333 \mathrm{abc}$ & 6143 & $4061 \mathrm{ab}$ & $4774 \mathrm{bcd}$ \\
\hline Chlorpyrifos/480 CE & 1.200 & 6461 & $7660 \mathrm{ab}$ & $6986 a b c$ & 6484 & 3677abcde & $458 \mathrm{lcd}$ \\
\hline Chlorpyrifos/10 G & 1.100 & 6051 & 7330abcd & $7107 \mathrm{abc}$ & 5583 & $3605 \mathrm{bcde}$ & $5035 \mathrm{abcd}$ \\
\hline Thiodicarb/350 TS & $700^{3}$ & 6627 & 7146abede & $7333 \mathrm{abc}$ & 4916 & $2967 \mathrm{fg}$ & $4687 \mathrm{~cd}$ \\
\hline Fipronil/20 G & 100 & 6433 & $7517 \mathrm{abc}$ & $7453 \mathrm{abc}$ & 5589 & 3575 cde & $4827 \mathrm{bcd}$ \\
\hline Fipronil/250 TS & $500^{3}$ & 6608 & 7016abcde & $7463 \mathrm{abc}$ & 6318 & $3385 \mathrm{cdef}$ & $4492 d$ \\
\hline Phorate/50 G & 2.500 & 6241 & $6823 \mathrm{cde}$ & $7019 \mathrm{abc}$ & 6113 & $4147 \mathrm{a}$ & $5372 \mathrm{abcd}$ \\
\hline Terbufos $/ 50 \mathrm{G}$ & 1.500 & 6003 & 7245abede & $7479 \mathrm{abc}$ & 6684 & 346 lcde & 5149 abcd \\
\hline Terbufos $/ 50 \mathrm{G}$ & 2.000 & 6682 & $6671 \mathrm{de}$ & $8016 a$ & 6795 & 3756abcd & $5737 \mathrm{abc}$ \\
\hline Imidacloprid/700 PM & $350^{3}$ & 6348 & & $8074 a$ & & $3609 \mathrm{bcde}$ & $5063 \mathrm{abcd}$ \\
\hline Imidacloprid/700 PM & $140^{3}$ & 6246 & & $7800 \mathrm{ab}$ & & $3411 \mathrm{cdef}$ & $6127 \mathrm{a}$ \\
\hline Tebupirimfos/20 G & 125 & 6253 & $7756 \mathrm{a}$ & $7429 \mathrm{abc}$ & 6152 & $3827 \mathrm{abc}$ & $4814 \mathrm{bcd}$ \\
\hline Furathiocarb/400 SC & $640^{3}$ & 6157 & & $6609 \mathrm{~cd}$ & & $2665 \mathrm{~g}$ & $4662 \mathrm{~cd}$ \\
\hline Isazofos $/ 50 \mathrm{SC}$ & 1.500 & 6127 & & $662 \mathrm{lcd}$ & & 377 labcd & $5123 \mathrm{abcd}$ \\
\hline Isazofos/50 SC & $1.500^{4}$ & 6293 & & $6774 \mathrm{bcd}$ & & $3220 \mathrm{ef}$ & 4958abcd \\
\hline Tarbofuran/50 G & 1.500 & 6350 & 7024abcde & $7109 \mathrm{abc}$ & 6004 & 3304 def & $5946 a b$ \\
\hline Tebupirimfos $/ 20 \mathrm{G}$ & 160 & & 7059abcde & & 6265 & & \\
\hline Tebupirimfos/20 G & 200 & & 6964 bcde & & 6088 & & \\
\hline Isazofos/50 SC & 500 & & $6594 \mathrm{de}$ & & 5363 & & \\
\hline Isazofos/50 SC & 750 & & $6667 \mathrm{de}$ & & 6514 & & \\
\hline Fsazofos/50 SC & 1.000 & & 7135abede & & 4864 & & \\
\hline Fipronil+thiodicarb/250+300 TS & $500+360^{3}$ & & $6509 \mathrm{c}$ & & 5914 & & \\
\hline C.V. $(\%)$ & & 10,7 & 3,9 & 10,7 & 8,5 & 9,7 & 16,9 \\
\hline
\end{tabular}

${ }^{1}$ Dados originais foram transformados para $\sqrt{ } \mathrm{X}+0,5$ para análise estatística.

${ }^{2}$ Médias seguidas da mesma letra, na coluna, não diferem significativamente, pelo teste de Tukey a 5\%. ${ }^{3} \mathrm{~g}$ i.a. $100 \mathrm{Kg}^{-1}$ sementes.

${ }^{4}$ Pulverização total da área.

Ano I: $\mathrm{L}_{1}, \mathrm{~L}_{2}=$ Batavo I e II, $\mathrm{L}_{3}=$ Castrolanda, $\mathrm{L}_{4}=$ Arapoti; Ano II: $\mathrm{L}_{1}=$ Castrolanda, $\mathrm{L}_{2}=$ Batavo.

dos valores de 2,5-3,00 obtidos em outros estudos referentes à perdas de rendimento. Mayo (1986) relatou que essa diferença pode ser atribuída à uniformidade da infestação conseguida com a infestação artificial realizada versus a variação encontrada nos trabalhos conduzidos sob infestação natural. Esses e outros estudos não estabelecem o nível de danos nas raízes que podem ser tolerados, mas evidenciam que danos nas raízes maiores que 3,0, na escala de 1 a 6, indicam perdas econômicas (Mayo, 1986). O dano na raiz e a redução do peso seco da parte aérea da planta de milho foram relacionados positivamente com a densidade de larvas no sistema radicular (Marques et al.,1999). Resultados obtidos por Davis \& Colleman (1997), utilizando terbufos para o controle de larva de Diabrotica, mostraram também variabilidade em anos alternados entre tratamento com inseticida e com rendimento de grãos.

\section{Conclusões}

1- Chlorpyrifos $10 \mathrm{G}$, terbufos $50 \mathrm{G}$, tebupirimfos $20 \mathrm{G}$, fipronil $20 \mathrm{G}$, na formulação granulada, e chlorpyrifos $450 \mathrm{SC}$ e $480 \mathrm{CE}$ aplicados e pulverizados, respectivamente, no sulco de plantio, foram os inseticidas que apresentaram o melhor controle da larva de Diabrotica spp.

2-Tratamento de sementes com thiodicarb, fipronil, imidacloprid, furathiocarb e fipronil+thiodicarb 
apresentou baixa performance no controle das larvas de Diabrotica.

3- A proteção eficiente dos inseticidas ao sistema radicular do milho proporcionou aumento significativo no rendimento de grãos.

\section{Literatura Citada}

APPLE, J. W.; CHIANG, H. C.; ENGLISH, L. M.; FRENCH, L. K.; KEASTER, A. J.; KRAUSE, G. F.; MAYO, Z. B.; MUNSON, J. D.; MUSICK, G. J.; OWENS, J. C.; RASMUSSEN, R. E.; SECHRIEST, J. J.; TOLLEFSON, J. J.; WEDBERG, J. L. Impact of northern and western corn rootworm larvae on field corn. Madison: University of Wisconsin, 1977. 10p. (North Central Regional Research Publication 239)

BERGMAN, M. K.; TOLLEFSON, J. J.; HINZ, P. N. Sampling scheme for estimating populations of corn rootworm larvae. Environmental Entomology, College Park, v.10, p.986-990, 1981.

BRANDENBURG, R. L.; HERBERT JR, D. A. Effect of timings on prophylatic treatments for Southern corn rootworm (Coleoptera: Chrysomelidae) in peanut. Journal of Economic Entomology, College Park, v.84, p. 1894-1898, 1991.

BRANSON, T. F.; ORTMAN, E. E. Host range of larvae of the northern corn rootworm: further studies. Journal of the Kansas Entomological Society, Lawrence, v.44, p 50-52, 1971.

BRANSON, T. F.; SUTTER, G. R.; FISHER, J. R. Plant response to stress induced by artificial infestations of western corn rootworm. Environmental Entomology, College Park, v.9, p. 253-257, 1980.

DAVIS, P. M. Comparison of economic injury levels for western corn rootworm (Coleoptera:
Chrysomelidae) infesting silage and grain corn. Journal of Economic Entomology, College Park, v.87, p. 1086-1090, 1994.

DAVIS, P. M.; COLEMAN, S. Managing corn rootworms: (Coleoptera Chrysomelidae) on dairy farms: the need for a soil insecticide. Journal of Economic Entomology, College Park, v.90, p. 205217, 1997.

GETZIN, L. W. Factors influencing the persistence and effectiveness of chlorpyrifos in the soil. Journal of Economic Entomology, College Park, v. 78, p. 412-414, 1985.

HARRIS, C. R. Further studies on the influence of soil moisture on the toxicity of insecticides in the soil. Journal of Economic Entomology, College Park, v 60. p. 41-44, 1967.

HARRIS, C. R. Influence of soil type on the activity of insecticides in soil. Journal of Economic Entomology, College Park, v 59, p. 1221-1225, 1966.

HILLS, T. M.; PETERS, D. C. A method of evaluating postplanting insecticide treatments for control of western corn rootworm larvae. Journal of Economic Entomology, College Park, v.64, p. 764-765, 1971.

LEE, S.; TSAO, R.; PETERSON, C.; COATS, J. $\mathrm{R}$. Insecticidal activity of monoterpenoids to western corn rootworm (Coleoptera: Chrysomelidae), twospotted spider mite (Acari: Tetranychidae), and house fly (Diptera: Muscidae). Journal of Economic Entomology, College Park, v.90,p. 883-892, 1997.

LEVINE, E.; OLOUMI-SADEGHI, H. Management of diabroticite rootworms in corn. Annual Review of Entomology, Palo Alto, v.36, p. 229-255, 1991. 
MARQUES, G. B. C.; ÁVILA, C. J.; PARRA, J. R. P. Danos causados por larvas e adultos de Diabrotica speciosa (Coleoptera: Chrysomelidae) em milho. Pesquisa Agropecuária Brasileira, Brasília, v. 34, n. 11, p. 1983-1986, 1999.

MAYO, Z. B. Field evaluation of insecticides for control of larvae of corn rootworms. In: KRYSAN, J. L.; MILLER, T. A. (Eds). Methods for the study of pest Diabrotica. New York: SpringerVerlag, 1986. p.183-203.

METCALF, R. L. Foreword. In: KRYSAN, J. L.; MILLER, T. A. eds. Methods for the study of pest Diabrotica. New York: Springer-Verlag, 1986. p.vii-xv.

SECHRIEST, R. E. Observations of the biology and behavior of corn rootworms. Proceedings North Central Branch Entomological Society of America , v.24, p.129-132, 1969.

SUTTER, G. R.; BRANSON, T. F.; FISHER, J. R.; ELLIOT, N. C.; JACKSON, J. J. Effect of insecticide treatments on root damage ratings of maize in controlled infestations of western corn rootworms (Coleoptera:Chrysomelidae). Journal of Economic Entomology, College Park, v.82, p. 1792-1798, 1989.
SUTTER, G. R.; BRASON, T. F.; FISHER, J. R.; ELLIOTT, N. C. Effect of insecticides on survival, development, fecundity, and sex ratio in controlled infestations of Western corn rootworn (Coleoptera: Chrysomelidae). Journal of Economic Entomology, College Park, v 84, p.1905-1912, 1991.

SUTTER, G. R.; FISHER, J. R.; ELLIOT, N. C.; BRANSON, T. F. Effect of insecticide treatments on root lodging and yield of maize in controlled infestations of western corn rootworms (Coleoptera: Chrysomelidae). Journal of Economic Entomology, College Park, v.83, p.2414-2420, 1990.

TURPIN, F. T.; DUMENIL, L. C.; PETERS, D. C. Decision-making related to use of soil insecticides by Indiana corn farmers. Journal of Economic Entomology, College Park, v.69, p. 359-362, 1972.

WEISS, M. J.; MAYO, Z. B. Potential of corn rootworm (Coleoptera: Chrysomelidae) larval counts to estimate larval populations to make control decisions. Journal of Economic Entomology, College Park, v.76, p. 158-161, 1983. 